\title{
A 'nice' bijection for a content formula for skew semistandard Young tableaux
}

\author{
Martin Rubey \\ Institut für Mathematik \\ Universität Wien \\ http://www . univie.ac.at/ ${ }^{\sim}$ rubey \\ a9104910@unet.univie.ac.at
}

Submitted: May 31, 2001; Accepted: April 14, 2002.

MR Subject Classifications: 05E10

\begin{abstract}
Based on Schützenberger's evacuation and a modification of jeu de taquin, we give a bijective proof of an identity connecting the generating function of reverse semistandard Young tableaux with bounded entries with the generating function of all semistandard Young tableaux. This solves Exercise 7.102 b of Richard Stanley's book 'Enumerative Combinatorics 2'.
\end{abstract}

\section{Introduction}

The purpose of this article is to present a solution for Exercise 7.102 b of Richard Stanley's book 'Enumerative Combinatorics 2' [5]. There, Stanley asked for a 'nice' bijective proof of the identity

$$
\sum_{\substack{R \text { reverse SSYT } \\ \text { of shape } \lambda / \mu \\ \text { with } R_{i j} \leq a+\mu_{i}-i}} q^{n(R)}=\left(\sum_{\substack{P \text { SSYT } \\ \text { of shape } \lambda / \mu}} q^{n(P)}\right) \cdot \prod_{\rho \in \lambda / \mu}\left(1-q^{a+c(\rho)}\right),
$$

where $a$ is an arbitrary integer such that $a+c(\rho)>0$ for all cells $\rho \in \lambda / \mu .{ }^{1}$ Here, and in the sequel, we use notation defined below:

Definition 1.1. A partition is a sequence $\lambda=\left(\lambda_{1}, \lambda_{2}, \ldots, \lambda_{r}\right)$ with $\lambda_{1} \geq \lambda_{2} \geq \cdots \geq \lambda_{r}>$ 0 , for some $r$.

\footnotetext{
${ }^{1}$ In fact, this is the corrected version of the identity originally given in [5], to be found at http://www-math.mit.edu/ rstan/ec. Stanley took it from [1], Theorem 3.1, where the formula is stated incorrectly, too.
} 


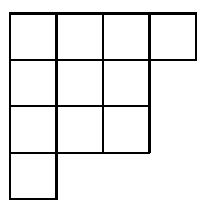

a. Ferrers diagram

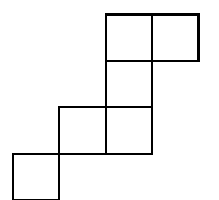

b. skew Ferrers diagram

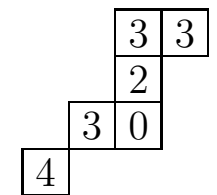

c. reverse SSYT

Figure 1.

The Ferrers diagram of a partition $\lambda$ is an array of cells with $r$ left-justified rows and $\lambda_{i}$ cells in row $i$. Figure 1.a shows the Ferrers diagram corresponding to $(4,3,3,1)$. We label the cell in the $i^{\text {th }}$ row and $j^{\text {th }}$ column of the Ferrers diagram of $\lambda$ by the pair $(i, j)$. Also, we write $\rho \in \lambda$, if $\rho$ is a cell of $\lambda$.

A partition $\mu=\left(\mu_{1}, \mu_{2}, \ldots, \mu_{s}\right)$ is contained in a partition $\lambda=\left(\lambda_{1}, \lambda_{2}, \ldots, \lambda_{r}\right)$, if $s \leq r$ and $\mu_{i} \leq \lambda_{i}$ for $i \in\{1,2, \ldots, s\}$.

The skew diagram $\lambda / \mu$ of partitions $\lambda$ and $\mu$, where $\mu$ is contained in $\lambda$, consists of the cells of the Ferrers diagram of $\lambda$ which are not cells of the Ferrers diagram of $\mu$. Figure 1.b shows the skew diagram corresponding to $(4,3,3,1) /(2,2,1)$. The content $c(\rho)$ of a cell $\rho=(i, j)$ of $\lambda / \mu$ is $j-i$.

Given partitions $\lambda$ and $\mu$, a tabloid of shape $\lambda / \mu$ is a filling $T$ of the cells of the skew diagram $\lambda / \mu$ with non-negative integers. $T_{\rho}$ denotes the entry of $T$ in cell $\rho$. The norm $n(T)$ of a tabloid $T$ is simply the sum of all entries of $T$. The content weight $w_{c}(T)$ of a tabloid $T$ is $\sum_{\rho \in \lambda / \mu} T_{\rho} \cdot(a+c(\rho))$, where $a$ is a given integer such that $a+c(\rho)>0$ for all cells $\rho \in \lambda / \mu$.

A semistandard Young tableau of shape $\lambda / \mu$, short $S S Y T$, is a tabloid $P$ such that the entries are weakly increasing along rows and strictly increasing along columns.

A reverse semistandard Young tableau of shape $\lambda / \mu$ is a tabloid $R$ such that the entries are weakly decreasing along rows and strictly decreasing along columns. In Figure 1.c a reverse SSYT of shape $(4,3,3,1) /(2,2,1)$ is shown.

\section{$2 \quad$ A Bijective proof of Identity 1}

In fact, we will give a bijective proof of the following rewriting of Identity 1 :

$$
\begin{aligned}
& \sum_{\substack{P \operatorname{SSYT} \\
\text { of shape } \lambda / \mu}} q^{n(P)}=\left(\sum_{\substack{R \text { reverse SSYT } \\
\text { of shape } \lambda / \mu \\
\text { with } R_{i j} \leq a+\mu_{i}-i}} q^{n(R)}\right) \cdot \prod_{\rho \in \lambda / \mu} \frac{1}{1-q^{a+c(\rho)}} \\
& =\sum_{\substack{(R, T) \\
R \text { reverse SSYT } \\
\text { of shape } \lambda / \mu}} q^{n(R)} q^{w_{c}(T)} \\
& \text { of shape } \lambda / \mu \\
& \text { with } R_{i j} \leq a+\mu_{i}-i \\
& \text { of shape } \lambda / \mu
\end{aligned}
$$


So all we have to do is to set up a bijection that maps $\operatorname{SSYT}^{\prime} \mathrm{x} P$ onto pairs $(R, T)$, where $R$ is a reverse SSYT with $R_{i j} \leq a+\mu_{i}-i$ and $T$ is an arbitrary tabloid, such that $n(P)=n(R)+w_{c}(T)$.

The bijection consists of two parts. The first step is a modification of a mapping known as 'evacuation', which consists of a special sequence of so called 'jeu de taquin slides'. An in depth description of these procedures can be found, for example, in Bruce Sagan's Book 'The symmetric group' [4], Sections 3.9 and 3.11. We use evacuation to bijectively transform the given SSYT $P$ in a reverse SSYT $Q$ which has the same shape and the same norm as the original one.

The second step of our bijection also consists of a sequence of - modified - jeu de taquin slides and bijectively maps a reverse SSYT $Q$ onto a pair $(R, T)$ as described above. This procedure is very similar to bijections discovered by Christian Krattenthaler, proving Stanley's hook-content formula. $[2,3]$

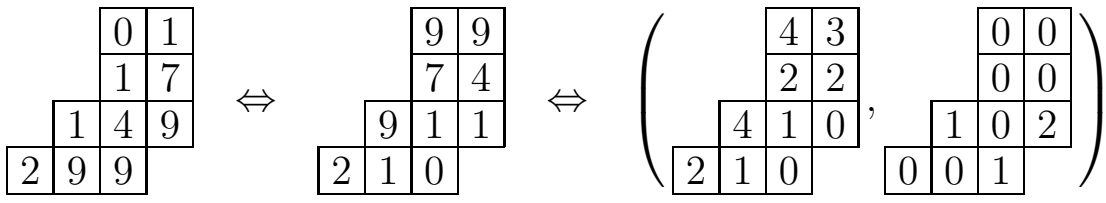

$$
\begin{aligned}
& n(.)=43 \quad n(.)=43 \quad n(.)=19, w_{c}(.)=24
\end{aligned}
$$

Figure 2.

A complete example for the bijection can be found in the appendix. There we chose $a=6$ and map the SSYT $P$ of shape $(4,4,4,3) /(2,2,1)$ on the left of Figure 2 to the reverse SSYT $Q$ in the middle of Figure 2, which in turn is mapped to the pair on the right of Figure 2, consisting of a reverse SSYT $R$, where the entry of the cell $\rho=(i, j)$ is less or equal to $a+\mu_{i}-i$, and a tabloid $T$ so that $n(Q)=n(R)+w_{c}(T)$.

In the algorithm described below we will produce a filling of a skew diagram step by step, starting with the 'empty tableau' of the given shape.

Theorem 2.1. The following two maps define a correspondence between SSYT'x and reverse SSYT'x of the same shape $\lambda / \mu$ and the same norm:

Given a SSYT $P$ of shape $\lambda / \mu$, produce a reverse SSYT $Q$ of the same shape and the same norm as follows:

Let $Q$ be the empty tableau of shape $\lambda / \mu$.

WHILE there is a cell of $P$ which contains an entry

Let $e$ be the minimum of all entries of $P$. Among all cells $\tau$ with $P_{\tau}=e$, let $\rho=(i, j)$ be the cell which is situated most right.

WHILE $\rho$ has a bottom or right neighbour in $P$ that contains an entry 
Denote the entry to the right of $\rho$ by $x$ and the entry below $\rho$ by $y$. We allow also that there is only an entry to the right or below $\rho$ and the other cell is missing or empty.

If $x<y$, or there is no entry below $\rho$, then replace

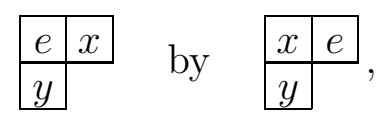

and let $\rho$ be the cell $(i, j+1)$.

Otherwise, if $x \geq y$, or there is no empty to the right, replace

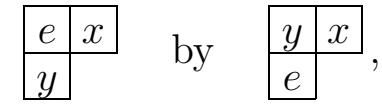

and let $\rho$ be the cell $(i+1, j)$.

END WHILE.

Put $Q_{\rho}$ equal to $e$ and delete the entry of the cell $\rho$ from $P$. Note that cells of $P$ which contain an entry still form a SSYT. In the proof below, $\rho$ will be called the cell where the jeu de taquin slide stops.

\section{END WHILE.}

$\ominus$ Given a reverse SSYT $Q$ of shape $\lambda / \mu$, produce a SSYT $P$ of the same shape and the same norm as follows:

Let $P$ be the empty tableau of shape $\lambda / \mu$.

WHILE there is a cell of $Q$ which contains an entry

Let $e$ be the maximum of all entries of $Q$. Among all cells $\tau$ with $Q_{\tau}=e$, let $\rho=(i, j)$ be the cell which is situated most left.

Set $P_{\rho}=e$ and delete the entry of the cell $\rho$ from $Q$.

WHILE $\rho$ has a top or left neighbour in $P$ that contains an entry

Denote the entry to the left of $\rho$ by $x$ and the entry above $\rho$ by $y$. We allow also that there is only an entry to the left or above $\rho$ and the other cell is missing or empty.

If $x>y$, or there is no entry above $\rho$, then replace

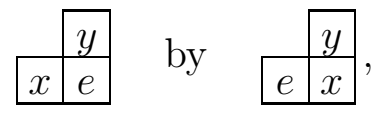

and let $\rho$ be the cell $(i, j-1)$.

Otherwise, if $x \leq y$, or there is no entry to the left, replace

$$
\begin{array}{|l|l|l|l|}
\cline { 2 - 3 } & y \\
\hline x & e
\end{array} \quad \text { by } \quad \begin{array}{ll}
\hline x \\
\hline
\end{array}
$$

and let $\rho$ be the cell $(i-1, j)$. 


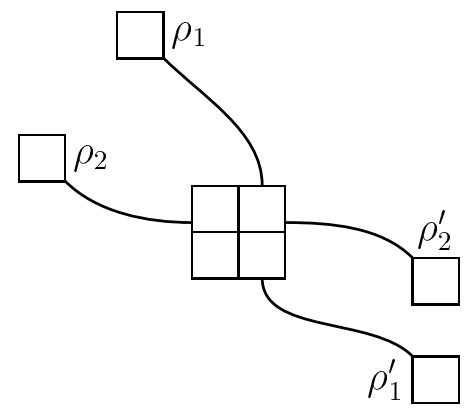

Figure 3.

\section{END WHILE.}

The cells of $P$ which contain an entry now form a SSYT. In the proof below, $\rho$ will be called the cell where the jeu de taquin slide stops.

\section{END WHILE.}

Proof. Note that what happens during the execution of the inner loop of $\ominus(\ominus)$ is a jeu de taquin forward (backward) slide performed on $Q$ into the cell $\rho$, see Section 3.9 of [4].

First we have to show that $\Theta$ is well defined. I.e., we have to check that after each jeu de taquin forward slide, after the entry $e$ in the cell $\rho$ is deleted from $P$, the cells of $P$ which contain an entry form a SSYT as stated in the algorithm. This follows, because after either type of replacement in the inner loop the only possible violations of increase along rows and strict increase along columns in $P$ can only involve $e$ and the entries to its right and below. When the jeu de taquin forward slide is finished, $\rho$ is a bottom-right corner of $P$, hence after deleting the entry in $\rho$ no violations of increase or strict increase can occur.

Next we show that $\ominus$ indeed produces a reverse SSYT. In fact, we even show that the tabloid defined by the cells of $Q$ which have been filled already, is a reverse SSYT at every stage of the algorithm.

Clearly, every cell of $Q$ is filled with an entry exactly once. Furthermore, at the time the cell $\rho$ is filled, the cells in $Q$ to the right and to the bottom of $\rho$-if they exist - are filled already, otherwise $\rho$ would not be a bottom-right corner of $P$. Because the sequence of entries chosen is monotonically increasing, rows and columns of $Q$ are decreasing.

So it remains to show that the columns of $Q$ are in fact strictly decreasing. Suppose that $\rho_{1}$ and $\rho_{2}$ are cells both containing the same minimal entry $e$, and $\rho_{1}$ is right of $\rho_{2}$.

When the jeu de taquin forward slide in $\ominus$ is performed into the cell $\rho_{1}$, the entry $e$ describes a path from $\rho_{1}$ to the cell where the slide stops, which we will denote by $\rho_{1}^{\prime}$. Similarly, we have a path from $\rho_{2}$ to a cell $\rho_{2}^{\prime}$.

Now suppose $\rho_{1}^{\prime}$ is in the same column as, but below $\rho_{2}^{\prime}$, as depicted in Figure 3. Clearly, in this case the two paths would have to cross and we had the following situation: 
First, (the star is a placeholder for an entry we do not know)

$$
\begin{array}{|l|l|}
\hline * & c \\
\hline z & y \\
\hline
\end{array} \quad \text { would be replaced by } \begin{array}{|l|l|}
\hline * & y \\
\hline z & c \\
\hline
\end{array}
$$

In this situation, $z$ would have to be smaller then $y$.

Then, when the jeu de taquin forward slide into the cell $\rho_{2}$ is performed, the following situation would arise at the same four cells:

$$
\begin{array}{|l|l|}
\hline c & y \\
\hline z & * \\
\hline
\end{array} \quad \text { would have to be replaced by } \begin{array}{|l|l|}
\hline y & c \\
\hline z & * \\
\hline
\end{array}
$$

But this cannot happen, because then $y$ would have to be strictly smaller than $z$.

It can be shown in a very similar manner that $\ominus$ indeed produces a SSYT. We leave the details to the reader.

Finally, we want to prove that $\Theta$ is inverse to $\Theta$. Suppose that in $\Theta$, a jeu de taquin forward slide into the cell $\rho$ containing the entry $e$ is performed on $P$. Suppose that the slide stopped in $\rho^{\prime}, Q_{\rho^{\prime}}$ is set to $e$ and the entry in $\rho^{\prime}$ is deleted from $P$. Among the entries of $Q, e$ is maximal, because smallest entries are chosen first in $\ominus$. Furthermore, among those cells of $Q$ containing the entry $e$, the cell $\rho^{\prime}$ is most left. This follows, because the tabloid defined by the cells of $Q$ which have been filled already, is a reverse SSYT, and the paths defined by the jeu de taquin slides cannot cross, as we have shown above.

It is straightforward to check that in this situation the jeu de taquin backward slide into $\rho^{\prime}$ performed on $P$ in $\Theta$ stops in the original cell $\rho$. By induction we find that $\Theta$ is inverse to $\ominus$.

The second step of the bijection is just as easy:

Theorem 2.2. The following two maps define a correspondence between reverse SSYT'x $Q$ to pairs $(R, T)$, where $R$ is a reverse $S S Y T$ with $R_{i j} \leq a+\mu_{i}-i$ and $T$ is an arbitrary tabloid, so that $n(Q)=n(R)+w_{c}(T), Q, R$ and $T$ being of shape $\lambda / \mu$ :

Given a reverse SSYT $Q$ of shape $\lambda / \mu$, produce a pair $(R, T)$ as described above as follows:

Set $R=Q$ and set all entries of $T$ equal to 0 .

WHILE there is a cell $\tau=(i, j)$ such that $R_{\tau}>a+\mu_{i}-i$

Let $e$ be maximal so that there is a cell $\tau$ with $R_{\tau}-(a+c(\tau))=e$. Among all cells $\tau$ with $R_{\tau}-(a+c(\tau))=e$, let $\rho=(i, j)$ be the cell which is situated most bottom. Set $R_{\rho}=e$.

WHILE $e<R_{(i, j+1)}$ or $e \leq R_{(i+1, j)}$

Denote the entry to the right of $\rho$ by $x$ and the entry below $\rho$ by $y$. We allow also that there is only a cell to the right or below $\rho$ and the other cell is missing. 
If $x-1>y$, or there is no cell below $\rho$, then replace
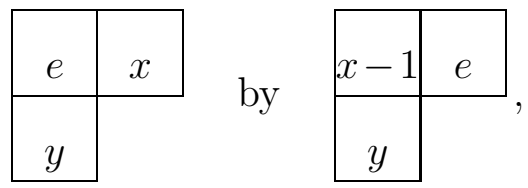

and let $\rho$ be the cell $(i, j+1)$.

Otherwise, if $y+1 \geq x$, or there is no cell to the right, replace
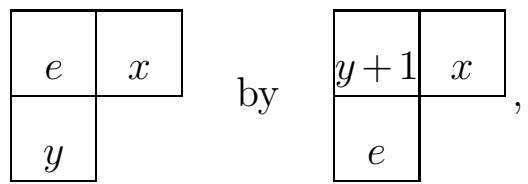

and let $\rho$ be the cell $(i+1, j)$.

END WHILE.

Increase $T_{\rho}$ by one.

END WHILE.

$\ominus$ Given a pair $(R, T)$ as described above, produce a reverse SSYT $Q$ of shape $\lambda / \mu$ as follows:

Set $Q=R$.

WHILE there is a cell $\tau=(i, j)$ such that $T_{\tau} \neq 0$

Let $e$ be minimal so that there is a cell $\tau$ with $Q_{\tau}=e$ and $T_{\tau} \neq 0$. Among these cells $\tau$ let $\rho=(i, j)$ be the cell which is situated most right. Decrease $T_{\rho}$ by one.

WHILE $e+a+c(\rho)>Q_{(i, j-1)}$ or $e+a+c(\rho) \geq Q_{(i-1, j)}$

Denote the entry to the left of $\rho$ by $x$ and the entry above $\rho$ by $y$. We allow also that there is only a cell to the left or above $\rho$ and the other cell is missing.

If $y>x+1$, or there is no cell above $\rho$, then replace

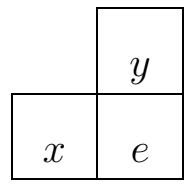

by

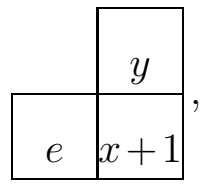

and let $\rho$ be the cell $(i, j-1)$.

Otherwise, if $x \geq y-1$, or there is no cell to the left, replace

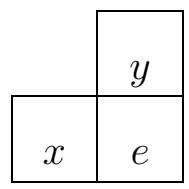

by

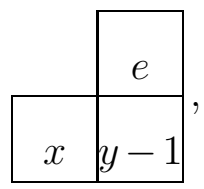

and let $\rho$ be the cell $(i-1, j)$. 


\section{END WHILE. \\ Increase $Q_{\rho}$ by $a+c(\rho)$. \\ END WHILE.}

Remark. Because of the obvious similarity to jeu de taquin slides, we will call what happens in the inner loop of $\Theta(\Theta)$ a modified jeu de taquin (backward) slide into $\rho$ performed on $R(Q)$.

Lemma 2.3. The two maps 2.2. $\ominus$ and 2.2. $\ominus$ are well defined. I.e. the tabloid $R$ produced by $\Theta$ is indeed a reverse SSYT with $R_{i j} \leq a+\mu_{i}-i$ and the tabloid $Q$ produced by $\Theta$ is indeed a reverse SSYT. Also, the equation $n(Q)=n(R)+w_{c}(T)$ holds.

Furthermore, the following statement is true: Suppose that $\ominus$ performs a modified jeu de taquin slide on $R$ into a cell $\rho_{1}$ with $R_{\rho_{1}}=e$. After this, suppose that another modified jeu de taquin slide on $R$ into a cell $\rho_{2}$ with the same entry $e$ is performed. Let $\rho_{1}^{\prime}$ and $\rho_{2}^{\prime}$ be the cells where the slides stop. Then $\rho_{1}^{\prime}$ is left of $\rho_{2}^{\prime}$ or $\rho_{1}^{\prime}=\rho_{2}^{\prime}$. A corresponding statement holds for Algorithm 2.2.ค.

Proof. First of all, we have to prove that Algorithm 2.2. $\ominus$ terminates. We required that $a+c(\tau)>0$ for all cells $\tau$, which implies that every time when we replace the entry in cell $\rho$ by $e$ (see the beginning of the outer loop of the algorithm) we decrease $\max _{\tau=(i, j)}\left(R_{\tau}-a-\mu_{i}+i\right)$. It is easy to see that this maximum is never increased in the subsequent steps of the algorithm.

It is easy to check that after every type of replacement within the modified jeu de taquin slides, the validity of the equation $n(Q)=n(R)+w_{c}(T)$ is preserved.

So it remains to show that after every modified jeu de taquin slide of $\Theta$, the resulting filling $R$ of $\lambda / \mu$ is in fact a reverse SSYT: We have that $Q_{\tau}-(a+c(\tau))=e$ is maximal at the very left of $\lambda / \mu$, because rows are decreasing in $Q$. Therefore, when $Q_{\tau}>a+\mu_{i}-i$, as required for the execution of the outer loop of $\Theta$, we have

$$
e=Q_{\tau}-(a+c(\tau))>a+\mu_{i}-i-\left(a+\mu_{i}+1-i\right)=-1,
$$

so $e$ is non-negative. Furthermore, after either type of replacement during the modified jeu de taquin slide, the only possible violations of decrease along rows or strict decrease along columns can involve only the entry $e$ and the entries to the right and below. By induction, $R$ must be a reverse SSYT.

The second statement of the lemma is shown with an argument similar to that used in the proof of Theorem 2.1.

When the jeu de taquin forward slide in $\ominus$ is performed into the cell $\rho_{1}$, the entry $e$ describes a path from $\rho_{1}$ to the cell $\rho_{1}^{\prime}$, where the slide stops. Similarly, we have a path from $\rho_{2}$ to $\rho_{2}^{\prime}$. We conclude that, if $\rho_{1}^{\prime}$ were strictly to the right of $\rho_{2}^{\prime}$, that these paths would have to cross. (See Figure 4). Hence we had the following situation:

First, (the star is a placeholder for an entry we do not know)

\begin{tabular}{|l|l|}
\hline$*$ & $z$ \\
\hline$e$ & $x$ \\
\hline
\end{tabular}

would be replaced by

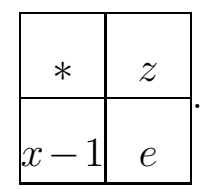




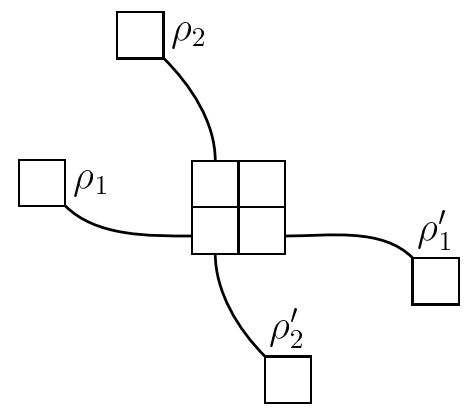

Figure 4.

In this situation, $x$ would have to be strictly smaller then $z$.

Then, when the modified jeu de taquin slide into $\rho_{2}$ is performed, the following situation would arise at the same four cells:

\begin{tabular}{|c|c|}
\hline$e$ & $z$ \\
\hline$x-1$ & $*$ \\
\hline
\end{tabular}

would have to be replaced by

\begin{tabular}{|l|l|}
\hline$x$ & $z$ \\
\hline$e$ & $*$ \\
\hline
\end{tabular}

But this cannot happen, because then $x$ would have to be at least as big as $z$ is.

The corresponding statement for Algorithm 2.2. $\ominus$ is shown similarly.

Proof of Theorem 2.2. It remains to show, that $\Theta$ and $\Theta$ are inverse to each other. This is pretty obvious considering the lemma:

Suppose that the pair $(R, T)$ is an intermediate result obtained after a modified jeu de taquin slide into the cell $\rho$. After this, $T_{\rho^{\prime}}$ is increased, where $\rho^{\prime}$ is the cell where the slide stopped. Then the entry in $\rho^{\prime}$ must be among the smallest entries of $R$, so that $T_{\rho^{\prime}} \neq 0$, because the sequence of $e$ 's in the cells chosen for the modified jeu de taquin slides is monotonically decreasing. If there is more than one cell $\rho$ which contains a minimal entry of $R$ and satisfies $T_{\rho} \neq 0$, the lemma asserts that the right-most cell was the last cell chosen for the modified jeu de taquin slide $\ominus$.

Hence it is certain that the right-most cell containing a minimal entry as selected before the modified jeu de taquin slide of $\Theta$ is $\rho^{\prime}$. It is easy to check, that the replacements done in $\ominus$ are exactly inverse to those in $\Theta$. For example, suppose the following replacement is performed in $\ominus$ :

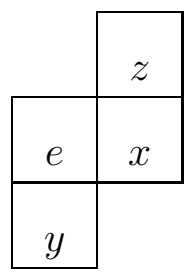

is replaced by

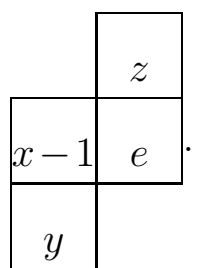

Then we had $x-1>y$ and, because of strictly decreasing columns, $z>x$. Therefore, in $\ominus$, this is reversed and we end up with the original situation.

Similarly, we can show that $\Theta$ is inverse to $\Theta$, too. 


\section{Appendix A: Step by step example}

This appendix contains a complete example for the algorithms described above for a SSYT of shape $(4,4,4,3) /(2,2,1)$ and $a=6$.

First the SSYT $P$ on the left of Figure 2 is transformed into the reverse SSYT $Q$ in the middle of Figure 2 using Algorithm 2.1. $\ominus$. The example has to be read in the following way: Each pair $(P, Q)$ in the table depicts an intermediate result of the algorithm. The cell of $P$ containing the encircled entry is the cell into which the next jeu de taquin slide is performed. The jeu de taquin path is indicated by the dotted line in $Q$.

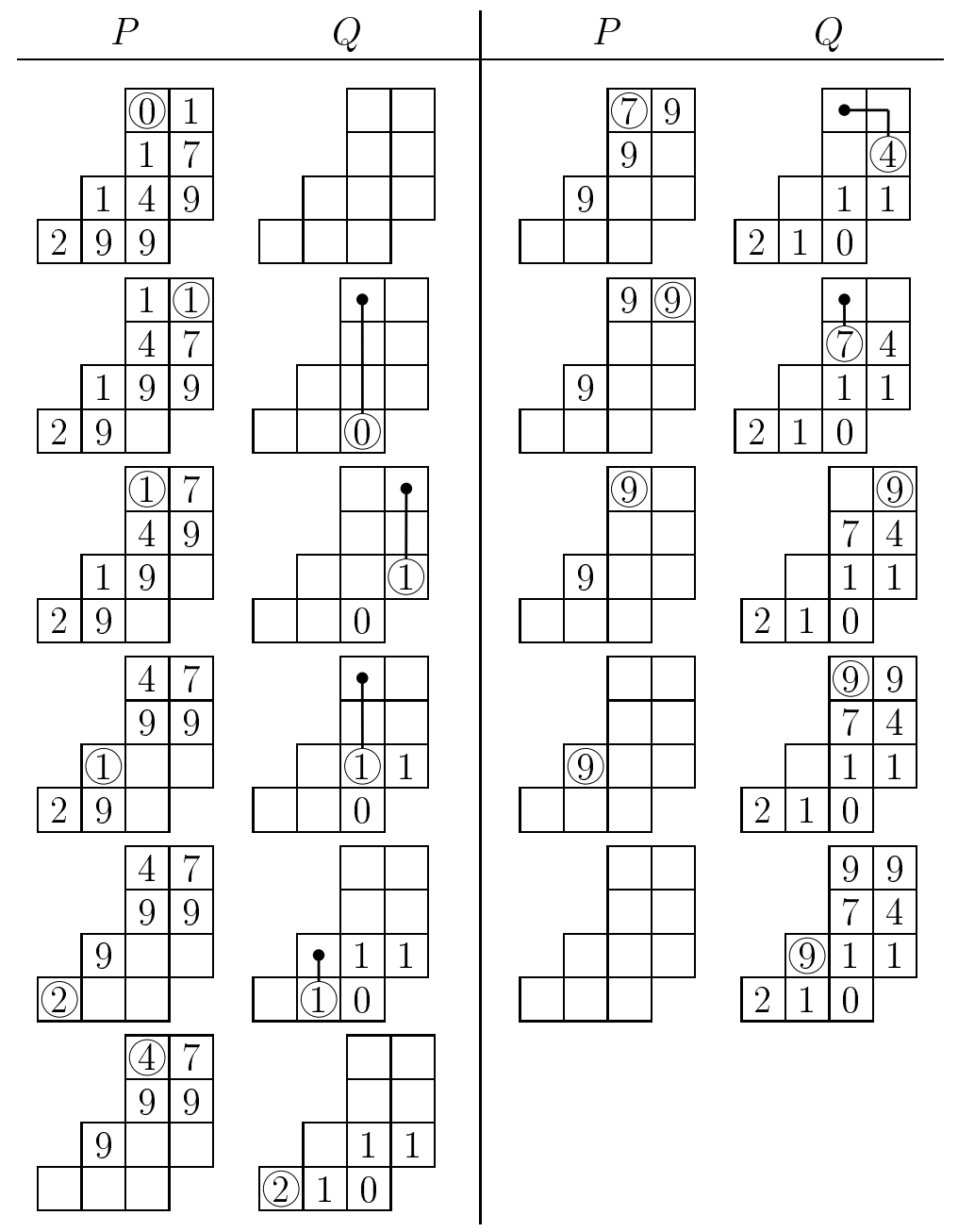

The inverse transformation $\Theta$ of the reverse SSYT $Q$ into the SSYT $P$ can be traced in the same table, we only have to start at the right bottom, where the tableau $P$ is empty, and work our way upwards to the top left of the table. Note that the jeu de taquin paths are the same.

In the second step of the bijection, this reverse SSYT $Q$ is mapped onto a pair $(R, T)$, where $R$ is a reverse SSYT with $R_{i j} \leq a+\mu_{i}-i, T$ is a tabloid and $n(Q)=n(R)+w_{c}(T)$.

First, the algorithm initialises $R$ to $Q$ and sets all entries of $T$ to zero. Using modified jeu de taquin slides, $R$ is then transformed into a reverse SSYT where the entries are 


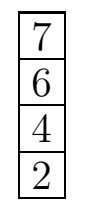

a. $a+\mu_{i}-i$

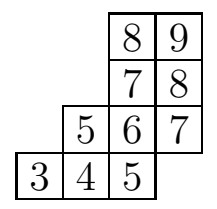

b. The tabloid with

entries $a+c(\rho)$

Figure 5 .

bounded as required. First the algorithm checks whether there are still cells in $R$ which are too large. For reference, we give the relevant bounds in Figure 5.a. Then, for selecting the cell into which the modified jeu de taquin slide is performed, we need to calculate $R_{\rho}-(a+c(\rho))$. Again, for reference we display these values for each cell in Figure 5.b.

Each row of the table below depicts an intermediate result of Algorithm 2.2. $\ominus$. The cells containing the encircled entry are the cells into which the modified jeu de taquin slide will be performed, the cells containing the boxed entry indicate, where the last modified jeu de taquin slide stopped. In the third column the jeu de taquin path for the selected cell is indicated.

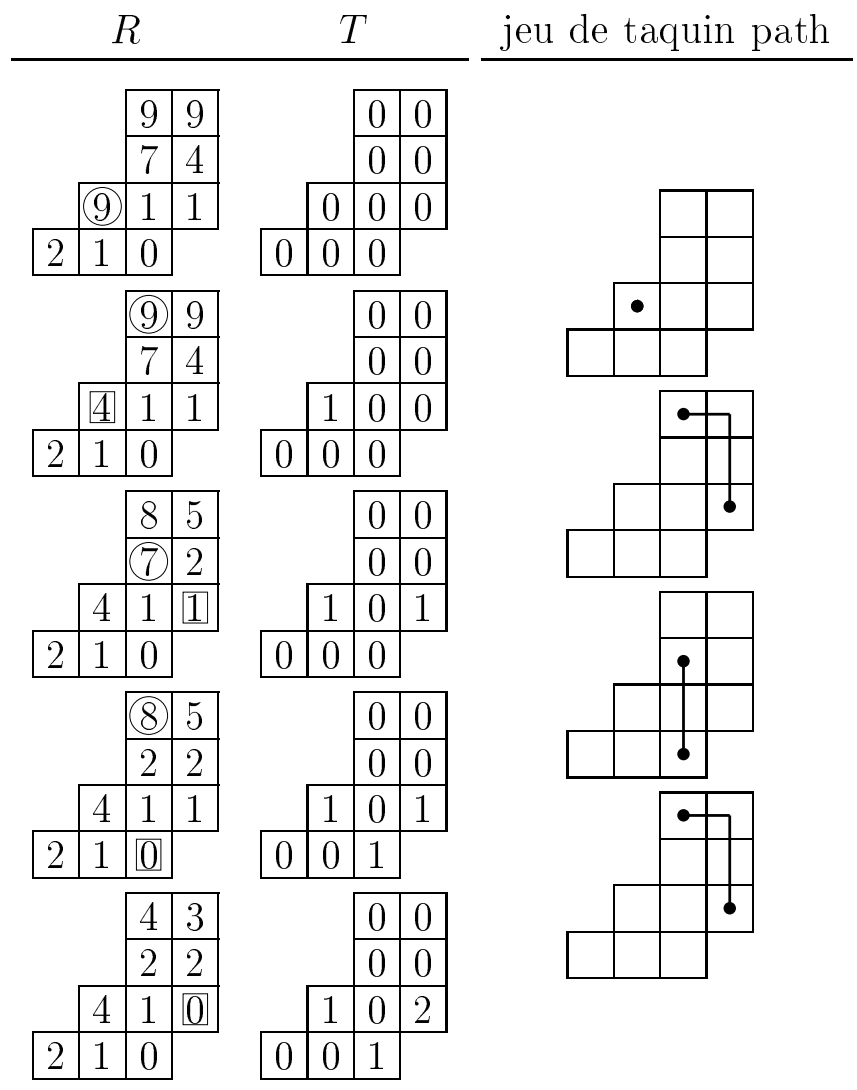

Again, the inverse transformation $\Theta$ can be traced in the same table, starting at the bottom, moving upwards. Now the cells containing the boxed entry are the cells into 
which the next modified jeu de taquin slide will be performed, the cells containing the encircled entry indicate where the last slide stopped. Of course, the jeu de taquin paths are the same as for $\Theta$.

\section{Appendix B: A complete matchup for SSYT'x of shape $(3,2) /(1)$ with norm 5 , where $a=2$}

In the table below you find a complete matchup for SSYT'x of shape $(3,2) /(1)$ with norm 5 where $a=2$. The first column contains all SSYT'x of shape $(3,2) /(1)$ and norm 5 . In the second column, the corresponding reverse SSYT'x obtained by evacuation are displayed. Finally, in columns three and four, the results of Algorithm 2.2. $\ominus$ can be found.

This table was produced with a Common-LISP-implementation of the algorithms above, which can be found on the author's homepage. ${ }^{2}$

\begin{tabular}{|c|c|c|c|}
\hline$P$ & $Q$ & $R$ & $T$ \\
\hline \begin{tabular}{|l|l|}
0 & 0 \\
\end{tabular} & \begin{tabular}{|l|l|}
5 & 0 \\
\end{tabular} & \begin{tabular}{|l|l|}
2 & 0 \\
\end{tabular} & \begin{tabular}{|l|l|}
1 & 0 \\
\end{tabular} \\
\hline \begin{tabular}{|l|l|}
0 & 5 \\
\end{tabular} & \begin{tabular}{|l|l|}
0 & 0 \\
\end{tabular} & \begin{tabular}{|l|l|}
0 & 0 \\
\end{tabular} & \begin{tabular}{|l|l|}
0 & 0 \\
\end{tabular} \\
\hline \begin{tabular}{|l|l|}
0 & 0 \\
\end{tabular} & \begin{tabular}{|l|l|}
4 & 0 \\
\end{tabular} & \begin{tabular}{|l|l|}
1 & 0 \\
\end{tabular} & \begin{tabular}{|l|l|}
1 & 0 \\
\end{tabular} \\
\hline \begin{tabular}{|l|l|}
1 & 4 \\
\end{tabular} & \begin{tabular}{|l|l|}
1 & 0 \\
\end{tabular} & \begin{tabular}{|l|l|}
0 & 0 \\
\end{tabular} & \begin{tabular}{|l|l|}
1 & 0 \\
\end{tabular} \\
\hline \begin{tabular}{|l|l|}
0 & 0 \\
\end{tabular} & \begin{tabular}{|l|l|}
3 & 0 \\
\end{tabular} & \begin{tabular}{|l|l|}
1 & 0 \\
\end{tabular} & \begin{tabular}{|l|l|}
0 & 0 \\
\end{tabular} \\
\hline \begin{tabular}{|l|l|}
2 & 3 \\
\end{tabular} & \begin{tabular}{|l|l|}
2 & 0 \\
\end{tabular} & \begin{tabular}{|l|l|}
0 & 0 \\
\end{tabular} & \begin{tabular}{|l|l|}
2 & 1 \\
\end{tabular} \\
\hline \begin{tabular}{|l|l|}
0 & 1 \\
\end{tabular} & \begin{tabular}{l|l|}
1 & 0 \\
\end{tabular} & \begin{tabular}{|l|l|}
1 & 0 \\
\end{tabular} & \begin{tabular}{|l|l|}
0 & 0 \\
\end{tabular} \\
\hline \begin{tabular}{|l|l|}
0 & 4 \\
\end{tabular} & \begin{tabular}{|l|l|}
4 & 0 \\
\end{tabular} & \begin{tabular}{|l|l|}
0 & 0 \\
\end{tabular} & \begin{tabular}{|l|l|}
4 & 0 \\
\end{tabular} \\
\hline \begin{tabular}{|l|l|}
0 & 1 \\
\end{tabular} & \begin{tabular}{|l|l|}
3 & 0 \\
\end{tabular} & \begin{tabular}{|l|l|}
2 & 0 \\
\end{tabular} & \begin{tabular}{|l|l|}
0 & 0 \\
\end{tabular} \\
\hline \begin{tabular}{|l|l|}
1 & 3 \\
\end{tabular} & \begin{tabular}{|l|l|}
1 & 1 \\
\end{tabular} & \begin{tabular}{|l|l|}
0 & 0 \\
\end{tabular} & \begin{tabular}{|l|l|}
1 & 1 \\
\end{tabular} \\
\hline \begin{tabular}{|l|l|}
0 & 1 \\
\end{tabular} & \begin{tabular}{|l|l|}
2 & 0 \\
\end{tabular} & \begin{tabular}{|l|l|}
2 & 0 \\
\end{tabular} & \begin{tabular}{|l|l|}
0 & 0 \\
\end{tabular} \\
\hline \begin{tabular}{|l|l|}
2 & 2 \\
\end{tabular} & \begin{tabular}{|l|l|}
2 & 1 \\
\end{tabular} & \begin{tabular}{|l|l|}
0 & 0 \\
\end{tabular} & \begin{tabular}{|l|l|}
1 & 1 \\
\end{tabular} \\
\hline \begin{tabular}{|l|l|}
0 & 2 \\
\end{tabular} & \begin{tabular}{|l|l|}
2 & 0 \\
\end{tabular} & \begin{tabular}{|l|l|}
2 & 0 \\
\end{tabular} & \begin{tabular}{|l|l|}
0 & 0 \\
\end{tabular} \\
\hline \begin{tabular}{|l|l|}
0 & 3 \\
\end{tabular} & \begin{tabular}{|l|l|}
3 & 0 \\
\end{tabular} & \begin{tabular}{|l|l|}
0 & 0 \\
\end{tabular} & \begin{tabular}{|l|l|}
3 & 0 \\
\end{tabular} \\
\hline \begin{tabular}{|l|l|}
0 & 2 \\
\end{tabular} & \begin{tabular}{|l|l|}
2 & 2 \\
\end{tabular} & \begin{tabular}{|l|l|}
2 & 2 \\
\end{tabular} & \begin{tabular}{|l|l|}
0 & 0 \\
\end{tabular} \\
\hline \begin{tabular}{|l|l|l}
1 & 2 \\
\end{tabular} & \begin{tabular}{|l|l|}
1 & 0 \\
\end{tabular} & \begin{tabular}{|l|l|}
0 & 0 \\
\end{tabular} & \begin{tabular}{|l|l|l}
1 & 0 \\
\end{tabular} \\
\hline
\end{tabular}

${ }^{2}$ http://www.mat.univie.ac.at/ ${ }^{\sim}$ rubey/biject.lisp 


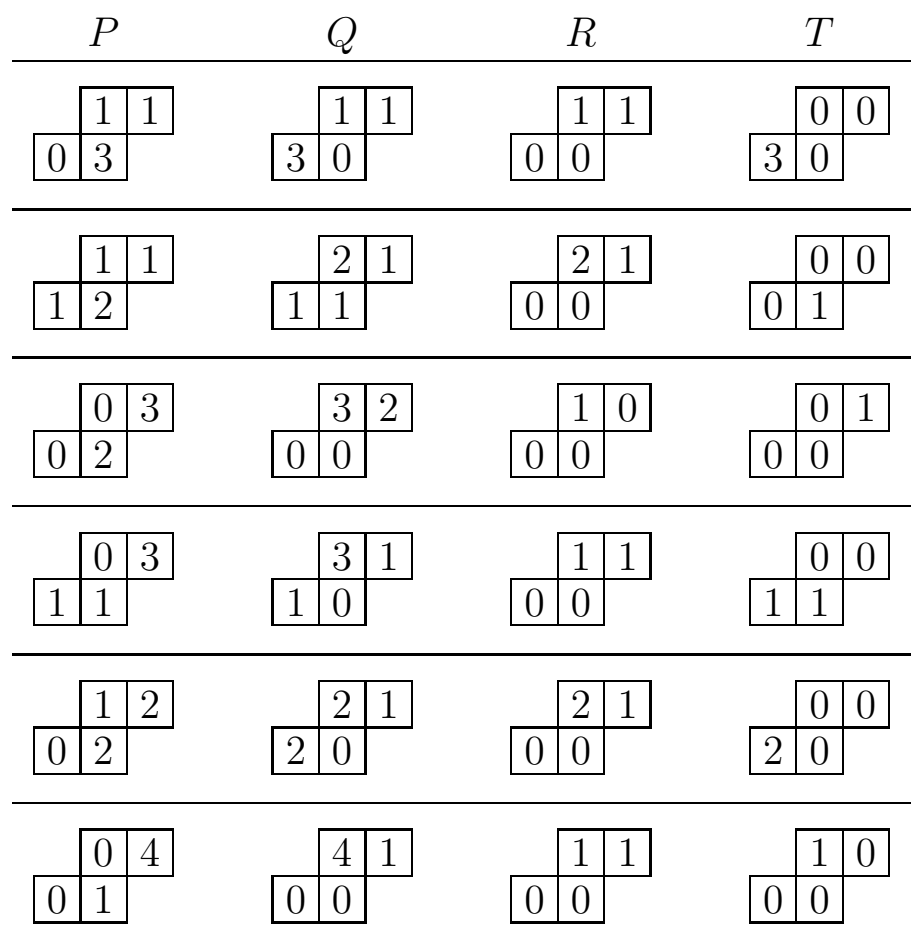

\section{References}

[1] Sara C. Billey, William Jockusch, and Richard P. Stanley, Some combinatorial properties of Schubert polynomials, Journal of Algebraic Combinatorics 2 (1993), no. 4, $345-374$.

[2] Christian Krattenthaler, An involution principle-free bijective proof of Stanley's hookcontent formula, Discrete Mathematics and Theoretical Computer Science (1998), no. $3,11-32$.

[3] _ Another involution principle-free bijective proof of Stanley's hook-content formula, Journal of Combinatorial Theory, Series A (1999), no. 88, 66-92.

[4] Bruce E. Sagan, The symmetric group, Wadsworth \& Brooks/Cole, Pacific Grove, California, 1987.

[5] Richard P. Stanley, Enumerative combinatorics, vol. 2, Cambridge University Press, 1999. 